\title{
Tuberculoma of Central Nervous System with Right Sided Hemiparesis
}

\author{
Shahed lqbal ${ }^{*}$ \\ Wahida Akter ${ }^{\dagger}$ \\ M Badruddoza' \\ Mahmood Ahmed Chowdhury \\ 'Institute of Child Health \\ Chattagram Maa-O-Shishu Hospital Medical College \\ Chittagong, Bangladesh.
}

\begin{abstract}
Tuberculoma is one of the manifestations of Central Nervous System (CNS) tuberculosis (TB). A tuberculoma is a tuberculous focus, which enlarges with in brain tissue, firm, avascular, spherical masses, with size varying between $2 \mathrm{~cm}$ to 10 $\mathrm{cm}$ in diameter and the compressed surrounding tissue shows edema and gliosis. Tuberculoma resuls from aggregation of caseous tubercle that usually manifest clinically as brain tumour. Tuberculomas account for upto $40 \%$ of brain tumours in some areas of the world. In adults tuberculomas are most often supratentorial, but in children they are often infratentorial, located at the base of the brain near the cerebellum. Lesions are most often singular but may be multiple. The most common symptoms are headache, fever \& convulsion $\&$ also may give rise to signs of raised intracranial pressure or a hemiplegia, or cranial nerve palsy if in the brain stem. Here described a case of 4 years old female child who presented with the complants of high grade fever for 10 days duration followed by right sided weakness for 4 months. She had $6^{\text {th }}$ and $7^{\text {th }}$ cranial nerve palsy with exaggerated reflex on right side. Her tuberculin test was positive but Cerebro Spinal Fluid (CSF) study was normal \& Magnetic Resonance Imaging (MRI) of brain showed rim enhancing lesion. Early recognition and timely treatment of CNS TB is critical if the considerable mortality and morbidity associated with the condition is to be prevented.
\end{abstract}

Key words: Tuberculoma; Meningitis; MRI; Computerized Tomography (CT) scan.

\section{INTRODUCTION}

Central nervous system tuberculosis is a serious form of tuberculosis, due to hematogenous spread of Mycobacterium tuberculosis. Manifesting as meningitis, cerebritis, \& tuberculous abscesses or tuberculomas, affecting disproportionately children \& immunocompromised patient. Other risk factors include malnutrition \& malignancies ${ }^{1}$. Central nervous system tuberculosis includes three clinical categories: tuberculous meningitis, intracranial tuberculoma \& spinal tuberculous arachnoiditis. All three forms of CNS infections are encountered in regions of the world where the incidence of TB is high \& the prevalence of post- primary dissemination is common among children $\&$ young adults ${ }^{2,3}$.

The clinical presentation of CNS tuberculoma is more subtle than that of TB meningitis \& may include headache, seizure, focal neurologic deficits \& papilledema ${ }^{4}$. Lesion may occur in the brain, spinal cord, subarachnoid, subdural, or epidural space; they may be solitary but are most often multiple \& accompanied by surrounding edema \& ring enhancement ${ }^{5}$. Tuberculomas \& meningitis may be present simultaneously in up to $10 \%$ of cases $^{6}$.
Dr. Shahed lqbal

Assistant Professor

Institute of Child Health

Chattagram Maa-O-Shishu Hospital Medical College Chittagong, Bangladesh.

Mobile : +88 01713162228

Email :dr.shahedmasud@gmail.com

http://www.banglajol.info/index.php/CMOSHMCJ 


\section{CASE HISTORY}

Ms. "A" is a 4 years old immunized girl, $1^{\text {st }}$ issue of a nonconsanguinous parent hailing from Laxmipur presented with the complaints of right sided weakness for last 4 months. She was completely well $41 / 2$ months back. Then she developed fever which was high grade intermittent in nature not associated with chills \& rigor. She was treated in local clinic by injectable antibiotic for 10 days. Fever subsided after 10 days, then she

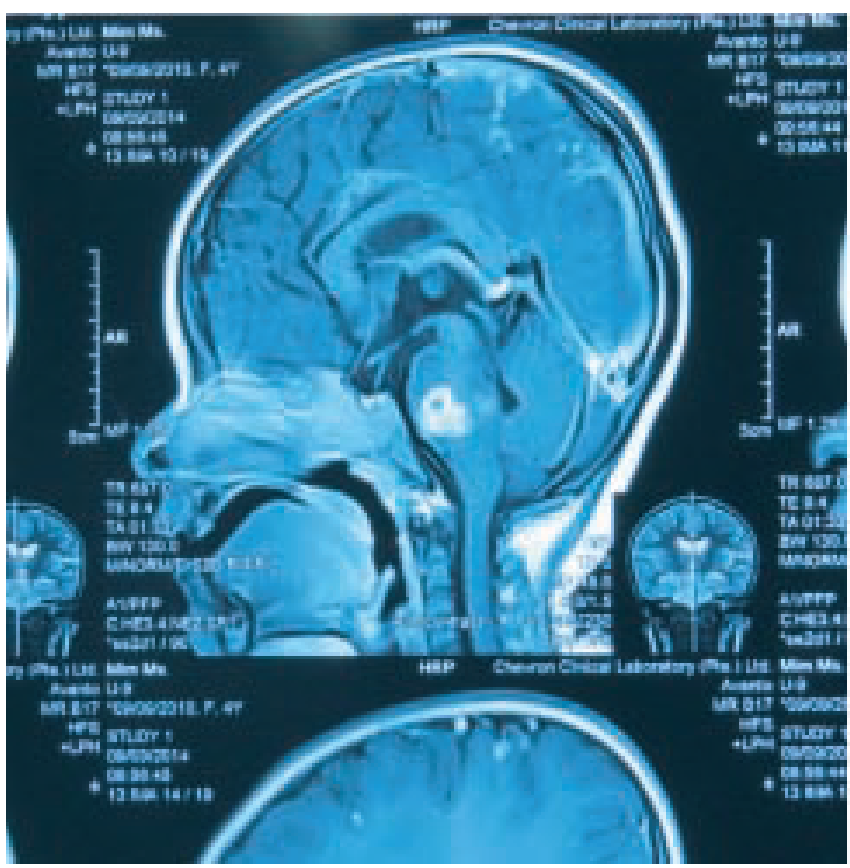

Figure 1
CXR: Opacity in both apical areas consistent with consolidation. Tuberculin test: positive (12 mm after 72 hour). Her CSF study was normal.MRI of brain showed multiple rim enhancing $T_{2}$ hypointense region in the mid pons, left side of upper pons and bilateral high frontoparietal sulcus with nonenhancing surrounding edema. Feature suggestive of multiple tuberculoma (Figure 1,2).

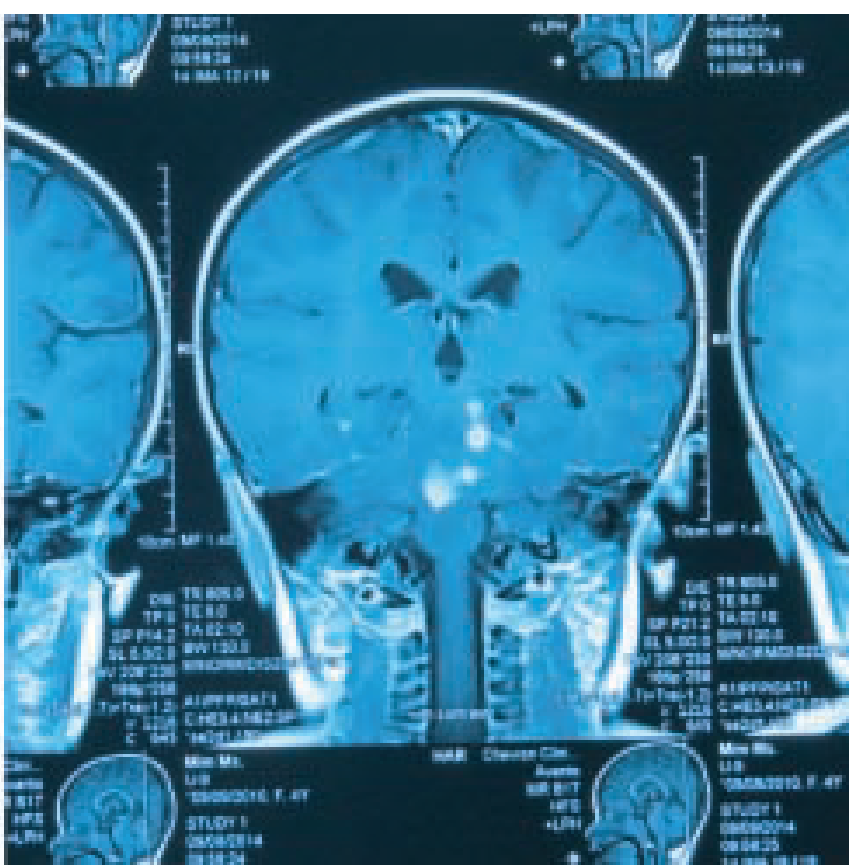

Figure 2

Figure 1 \& 2: MRI of brain shows multiple rim enhancing $\mathrm{T}_{2}$ hypointense region in the mid pons, left side of upper pons and bilateral high frontoparietal sulcus with nonenhancing surrounding edema

developed right sided weakness involving right side of face, right upper \& lower limb. Initially she can walk with limping, later she used to fall frequently with attempt of walk. She was treated locally for her weakness but the condition didn't improved. There was no history of headache, vomiting, seizure or head trauma. Mother had history of pulmonary TB 5 years back \& she completed her treatment 1 year before the birth of this child. Her bowel \& bladder history was uneventful.

On examination she was conscious, co-operative and normal vital signs. Skin survey revealed B C G mark. Back \& spine examination showed no abnormality. All cranial nerves were intact but there were right $6^{\text {th }} \& 7^{\text {th }}$ cranial nerve palsy. There were dropping of right side of face, loss of nasolabial fold on right side, wrinkling of forehead present in both sides. Her taste sensation was intact. Tone $\&$ power of muscle of right side was decreased, jerks were exaggerated on right side with plantar extensor on right side. Sensory examination was intact $\&$ there was no sign of meningeal irritation. She could walk with limp. Ophthamoscopic examination of eye revealed no abnormality. Examination of other system revealed no abnormality. $\mathrm{Hb} \%$ : $9.6 \mathrm{gm} / \mathrm{dl}$, ESR: $60 \mathrm{~mm}$ in $1^{\text {st }}$ hour, WBC: $11300 / \mathrm{mm}^{3}$, PLATELET: 4, 50000/mm³ , N: 60\%, L: 32\%, E: 4\%, M: 4\%,

\section{DISCUSSION}

CNS tuberculomas are rare but serious form of tuberculosis due to the haematogenous spread of Mycobacterium tuberculosis. Intracranial tuberculomas are the least common presentation of CNS TB, found only in $1 \%$ of patients ${ }^{6}$. Symptoms \& radiologic features are nonspecific, leading sometimes to misdiagnosis. This patient presented with history of high grade fever of 10 days duration with no history of headache, vomiting, seizure \& no history of TB contact. The bacilli reach the CNS by the hematogenous route secondary to disease elsewhere in the body. Chest X-ray of this patient shows bilateral apical opacities which may be consistent with tuberculous consolidation. The original description by them suggested a two stage development of CNS tuberculosis- first, tuberculous lesions (Rich's focus) develop in the brain during the stage of bacteremia or shortly afterward and later the rupture or growth of one or more of those lesions produces development of CNS tuberculosis ${ }^{8}$. The initial Rich focus may occur in the meninges, the subpial or subependymal surface of the brain, and may indeed remain dormant for many years. The specific stimulus needed for rupture or growth of these lesions is still not completely understood although the mechanism is known to be immunological in nature? 
Tuberculomas are thought to arise when tubercles in the brain parenchyma enlarge without rupturing into the subarachnoid space. As such, they often occur in the absence of Tubercular Meningitis (TBM) but certainly may occur along with TBM. They commonly arise as solitary lesion, but multiple in only 15 to $33 \%$ of the $\operatorname{cases}^{10}$. This patient had multiple lesion distributed in pons, frontal \& parietal lobe. Tuberculomas of brain show a typical granulomatous reaction consisting of epitheloid cells and giant cells mixed with predominantly lymphocytes around a central area of necrosis contains clear or straw coloured fluid, as opposed to pus ${ }^{11}$. Tuberculomas often present with symptoms \& sign of focal neurological deficit without evidence of systemic disease ${ }^{12}$.
The radiologic features are also nonspecific and differential diagnosis includes malignant lesions, sarcoidosis, pyogenic abscess, toxoplsmosis, and cysticercosis [1,12]

\section{CONCLUSION}

Early recognition and timely treatment of CNS TB is critical if the considerable mortality and morbidity associated with the condition is to be prevented. Due to its rarity, nonspecific symptoms and $\mathrm{X}$-ray findings, intracranial tuberculomas remain a clinical difficulty. For diagnosis of CNS tuberculoma $\mathrm{MRI} / \mathrm{CT}$ scan is essential which is not available in most areas of our country beyond the cities.

\section{DISCLOSURE}

All the authors declared no competing interest.

\section{REFERENCES}

1. Rock, R.B., Olin, Baker, C.A., Molitor, T.W., and Peterson, P.K. Central nervous system Tuberculosis: pathogenesis and clinical aspects. Clinical Microbiology Reviews. 2008; 21: 243-261.

2. Center for disease control and prevention: Trends in tuberculosis - United States, 2012.MMWR 2013, 62:201-205.

3. European Center for Disease control and prevention: Annual epidemiological report 2012.In reporting on 2010 surveillance data and 2011 epidemic intelligence data. Stockholm: ECDC; 2013

4. Garcia-Monco JC. Central nervous system tuberculosis.Neurol Clin. 1999; 17:737-759.

5. Bernaerts A, Vanhoenacker FM, Parizel PM, et al. Tuberculosis of the central nervous system:overview of neuroradilogical findings. Eur Radiol. 2003; 13:1876-1890.

6. Cruz AT, Starke JR. Clinical manifestation of tuberculosis in children. Paediatric Respiratory Re- views. 2007.8:107-117.

7. Pimentel, M.L.V.,Alves, S.M.V.,Novis,S.A.P., Brandao, R.Z., and Neto, E.B. Intracranial tuberculomas developing during treatment of pulmonary tuberculosis: case report. Arq NEUROPSIQUIATRI.2000; 58:572-577.

8. Rich AR, McCocdock HA. The pathogenesis of tuberculous meningitis. Bull Johns Hopkins Hosp.1933; 52:5-37.

9. Bermudez LE, Sangari FJ.Cellular and molecular mechanism of internalization of mycobacteria by host cells.Microbes Infect. 2001; $3: 37-42$.

10. Hejazi, N. and Hassler, W. Multiple intracranial tuberculomas with atypical response to tuberculostic chemotherapy: literature review and a case report. Infection. 1997; 25:41-46.

11. Kumar, R.,C.K.Pandey, N.Bose, and S.Sahay. Tuberculosis brain abscess: clinical presentation, pathophysiology and treatment (in children).Childs Nerv. Syst. 2002; 18:118-123.

12. Sahaiu- Srivastava, S. and Jones, B. Brainstem tuberculoma in the immunocompetent: case report and literature review. Clinical Neurology and neurosurgery. 2008; 110:302-304. 